\title{
A new laser method for cleaning micropalaeontological specimens
}

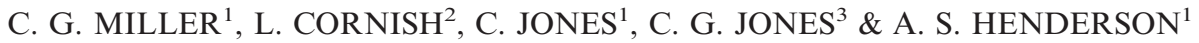 \\ ${ }^{1}$ Department of Palaeontology, The Natural History Museum, Cromwell Road, London SW7 5BD, UK. \\ ${ }^{2}$ Palaeontology Conservation Unit, The Natural History Museum, Cromwell Road, London SW7 5BD, UK. \\ ${ }^{3}$ Department of Mineralogy, The Natural History Museum, Cromwell Road, London SW7 5BD, UK (Current address: \\ Hitachi High Technologies, 7 Ivanhoe Road, Hogwood Industrial Estate, Finchampstead, Wokingham, Berks RG40 4QQ, UK).
}

\begin{abstract}
A Q-Switched Nd: YAG (neodymium yttrium aluminium garnet) infrared laser can be used to clean micropalaeontological specimens, particularly those coated in gold-palladium for SEM studies. Variable pressure SEM images taken of uncoated specimens before and after laser treatment show that the laser does not have a detrimental affect on micropalaeontological specimens composed of phosphate, silica or calcite in a number of wall structural forms. The laser has no effect on the textural surface of the specimen but flakes and crinkles water-soluble mounting glues used to fix specimens to the stub. Sticky carbon tabs (Reference Agar Scientific) were found to be the best mounting medium for holding specimens in place during treatment but microfossils were prone to become detached during the process if not attached firmly. Laser cleaning has a number of advantages over traditional methods of gold removal using sodium cyanide, which is toxic, slow and does not effectively remove gold-palladium coatings due to the insolubility of palladium in the reagent. This laser removal method has potential for removing matrix from specimens as well as other types of coating and mounting media including aluminium, carbon and wax. Safe removal of coatings releases important scientific information from gold-palladium coated museum micropalaeontological specimens. J. Micropalaeontol. 23(2): 165-169, November 2004.
\end{abstract}

\section{INTRODUCTION}

The scanning electron microscope is now the most commonly used method for illustrating micropalaeontological material in publications or reports. To view microfossils using a scanning electron microscope, it is often necessary to apply a thin coating of gold or gold-palladium ( $\mathrm{Au}-\mathrm{Pd})$ to specimens to create a conductive pathway. Other coatings such as carbon, aluminium, platinum and silver have also been used but are applied less commonly. However, some museums actively discourage any sort of coating of their specimens while on loan, as they often prove very difficult to remove. Scientific information about the specimens can also be lost as coatings obscure traces of internal structures and colours. It is possible to remove gold from specimens using a solution of sodium cyanide but there are health and safety concerns with this method and specimens have occasionally disintegrated. Another common coating, goldpalladium, looks similar to gold coating on micropalaeontological specimens, but cannot be removed reliably using the sodium cyanide method. Specimens in museums and research collections often do not have records of which type of coating has been used, particularly if the coatings were applied before the specimens were donated. A safer and potentially less destructive method is therefore needed to remove all types of coatings from these specimens. Gold coatings have been removed successfully by laser from a small shrew's jaw coated for variable pressure SEM study (Cornish \& Jones, 2002). Loose material on the surface of specimens has also been removed using this method. The same method is employed here on a variety of micropalae-

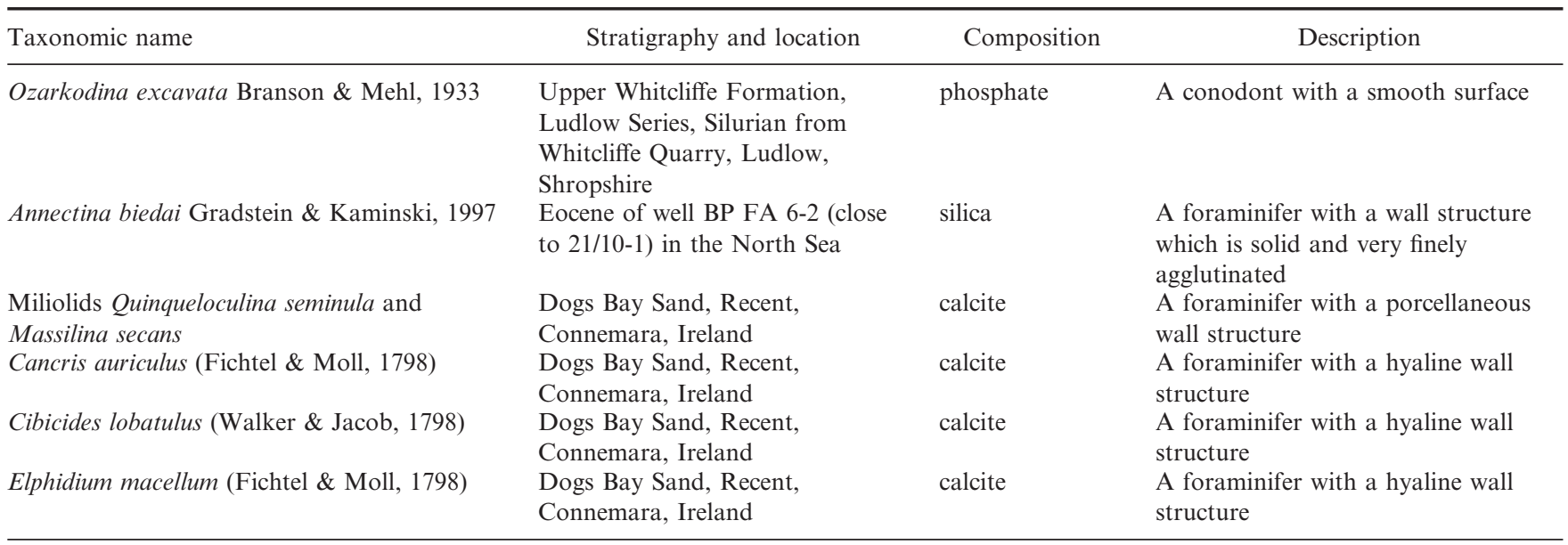

Table 1. Microfossil taxa used in the study. 

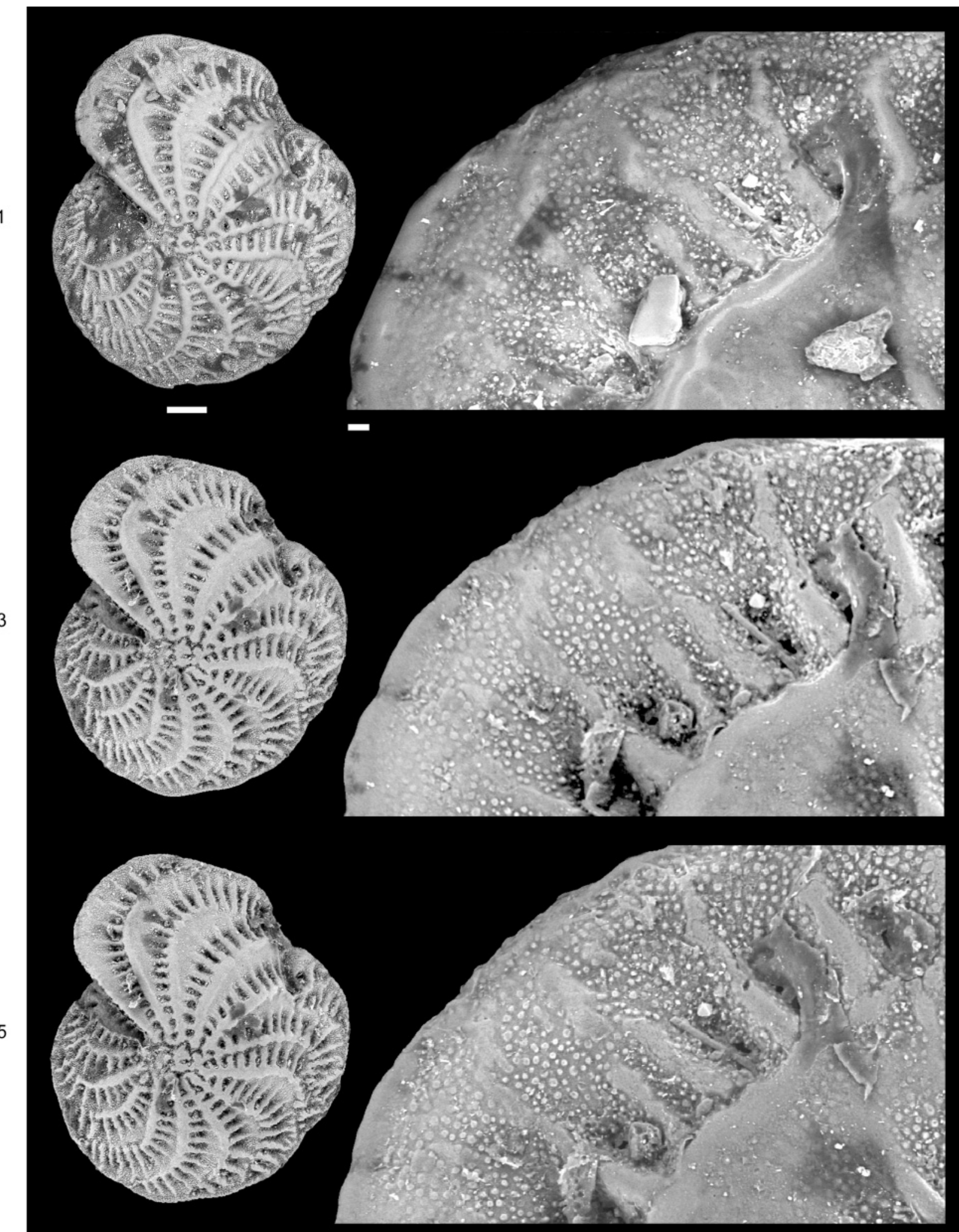
ontological specimens coated in gold-palladium to test the suitability of this method for micropalaeontologists and to test cleaning of specimens.

\section{TRADITIONAL METHODS FOR REMOVING SEM COATINGS}

Both the composition of the coating and, to a lesser extent, the nature and composition of the specimen determine exactly which of the more traditional methods for removing SEM coatings from micropalaeontological specimens is employed. Rather than describing exact methodologies here, the difficulties associated with some of these processes are highlighted.

Most of the micropalaeontological material imaged over the last twenty years at The Natural History Museum, London has been coated for SEM work with gold-palladium. Less commonly used now, though often found on specimens stored on stubs from earlier studies, is pure gold. Alternative coating materials include silver, aluminium and, occasionally, other pure or alloyed metals, as well as carbon and platinum. Metals, such as silver and aluminium, although providing good results in the SEM, are less commonly used, as silver tarnishes and aluminium oxidizes readily. As a result, they have to be removed as soon as possible after SEM work, as advanced tarnishing or oxidation renders the coating more difficult to remove and puts the specimen at greater risk of damage during the removal process.

In most cases, methods for removing these different types of coating involve either the use of highly toxic chemicals, or they are time consuming. This is especially true for metal coatings, although carbon, and some metals to a limited extent, can be removed by non-chemical means with a plasma asher or reverse polarity sputter-coater (Golden, 1989). The method most commonly employed for removing pure gold involves immersing the coated specimen in a weak solution of sodium cyanide and distilled water (Sela \& Boyde, 1977; Hodgkinson, 1991; Green, 2001). Even in concentrations of less than $10 \%$, this is a very toxic substance -raising health and safety concerns, not just during the coating removal process, but also in the safe disposal of waste and secure storage of the reagent. While this cyanide method provides excellent results for the removal of pure gold, in the authors' experience it has offered only limited success in removing the gold alloy, gold-palladium, although others (Golden, 1989) have cited better results. Alternatively, a 10\% ferric chloride $\left(\mathrm{FeCl}_{3}\right)$ mixture in ethanol may be used on $\mathrm{Au}-\mathrm{Pd}$ (Crissman \& McCann, 1979). Ferric chloride is light sensitive, requiring additional care during use and storage. Other methods include the use of aqua regia, a dilute mixture of hydrochloric and nitric acids. While this is effective on organic material such as palynomorphs it is, unfortunately, harmful to most inorganic material and, therefore, most types of microfossil. A weak solution of sodium hydroxide is used for the removal of aluminium coatings but this process needs to be monitored carefully to avoid excessive etching of specimens.

\section{THE LASER METHOD}

The term 'laser' is an acronym for 'light amplification by the stimulated emission of radiation'. The light produced is monochromatic, the beam is highly coherent and it can be focused to different spot sizes. The available wavelengths range from ultraviolet to infrared. The laser most commonly used to clean museum objects at present is the Q-switched Nd: YAG (neodymium yttrium aluminium garnet) laser operating at $1064 \mathrm{~nm}$ (Cooper, 1998). When the laser beam meets a boundary between two media, e.g. dirt and the sample surface, a proportion of the beam's energy is reflected, part is absorbed and the rest is transmitted. The proportion of energy absorbed is determined by the wavelength of the radiation (primary source) and the composition and molecular structure of the material. In order to remove unwanted surface coatings from an object it is important that the dirt or coating absorbs much more strongly than the underlying object at the selected laser wavelength. The coating absorbs the energy and is vapourized while the beam's energy is reflected from the underlying cleaned surface. A Q-switched laser is found to be very effective for cleaning natural history samples (Cornish \& Jones, 2002), as it acts as a high-speed shutter, which shortens the pulse length of the laser. This results in an extremely intense pulse of energy with very short pulse duration. The short pulse length ensures little or no temperature rise in the underlying surface and, therefore, less risk of thermal damage.

\section{MATERIALS}

Table 1 lists the microfossil taxa that were chosen for this study to reflect a wide variety of compositions, ages, wall structures and textures.

\section{METHODS}

One specimen of each of the microfossil types listed in Table 1 was fixed onto a $12.5 \mathrm{~mm}$ aluminium stub. The specimens were placed in a cross configuration in order to maximize the distance between them, with the outer specimens placed close to the edge so that each specimen was at least $5 \mathrm{~mm}$ away from its neighbour. Five different commonly used mounting methods for SEM were tested; dilute water soluble animal glue Gloy ${ }^{(\mathbb{R})}$ directly applied onto the stub, dilute PVC directly applied onto the stub, inverted photographic paper, Agar Scientific double-sided sticky carbon tabs and Araldite ${ }^{\circledR}$ epoxy resin. Acetone soluble adhesive was not tested as the Electron Microscope Unit at The Natural History Museum discourages its use. It is considered

Explanation of Plate 1.

Variable pressure backscattered SEM images of specimen NHM PM ZF 5158, Elphidium macellum (Fichtel \& Moll, 1798) from Dogs Bay Sand, Recent, Connemara, Ireland. Scale bars $100 \mu \mathrm{m}$ for all larger images and $10 \mu \mathrm{m}$ for close-up images. fig. 1. Specimen before gold-palladium coating and laser treatment. Note the dark areas of dirt particularly around the aperture. fig. 2. Close up of area above the aperture. Note the large pieces of loose dirt on the specimen and the ridge of glue running between them. fig. 3. Same specimen once gold-palladium had been applied and removed once. Note that the dirt around the aperture has been removed. fig. 4. Close up of same area as figure 2. Note that the large pieces of dirt have now been removed and the ridge of glue running between them has been reduced. fig. 5. Same specimen after gold-palladium has been applied and removed twice. Note that superficially the specimen looks the same as after one treatment (fig. 3). fig. 6. Close up of same area as figures 2 and 4. Note that the granular sculpture on the margin is now visible much more clearly (cf. fig. 4) and that there are still some remnants of glue in the top right-hand corner of the image (darker areas). 
best practice to avoid volatiles from the glue being pumped into the SEM column and contaminating the apertures, resulting in poor resolution. A control stub was prepared by mounting five miliolids in the same cross configuration using Gloy ${ }^{\mathbb{R}}$. All the mounted specimens were then imaged uncoated using a LEO 1455VP variable pressure SEM and a solid-state backscattered electron (BSE) detector. This imaging method was chosen as particles of dirt and any remaining coating can be seen clearly on the BSE SEM images (P1. 1). Carefully selected areas of the specimens were also imaged at higher magnification so that the surface texture could be seen clearly and compared after laser treatment. All specimens were then coated with $20 \mathrm{~nm}$ of gold-palladium using a Cressington 208HR sputter coater. The individual specimens on the control stub were treated with laser pulses at different wavelengths to determine the optimum wavelength to treat the other stubs. For each specimen, the laser was delivered by a hand-held articulated arm and aligned through a $5 \mathrm{~mm}$ diameter hole cut in a piece of thick card to prevent the laser from coming into contact with more than one specimen at a time. The fluence (energy density) was calculated for each laser treatment session. This is a measurement of energy per pulse divided by the surface area (spot size) of the laser beam measured in joules per square centimetre. The spot size was calculated by directing the laser at a piece of photographic paper and measuring the area affected. Each specimen on each stub was then treated with one pulse at the optimum wavelength to investigate the efficiency of removing the gold-palladium from specimens of differing composition and surface texture. These areas were then re-photographed in the variable pressure SEM using the same microscope conditions and magnifications. This enabled a comparative study of sample surfaces to be made both before and after laser treatment. Selected specimens were then re-coated and the gold-palladium removed by laser to check whether repeated laser treatments had any detectable affect (Pl. 1).

\section{RESULTS}

The control stubs showed that a wavelength of $1064 \mathrm{~nm}$ worked best to remove the gold-palladium coatings. Cleaning tests were also carried out using a visible green wavelength of $532 \mathrm{~nm}$, but little or no surface removal took place. The coatings were removed completely by treating with five pulses of the laser through the $5 \mathrm{~mm}$ mask. Optimum fluence (energy density) was calculated as $0.3 \mathrm{~J} \mathrm{~cm}^{-2}$. With specimens mounted on carbon tabs, some of the carbon was also removed from around the specimens and, in some cases, the aluminium stub could be clearly seen around the specimen. The best method involved suspending the cardboard mask using a clamp to prevent it from touching and damaging the specimens on the surface of the stub. All the specimens mounted in Araldite ${ }^{\mathbb{R}}$ and all but one of the specimens on the sticky carbon tabs survived the cleaning process. Specimens mounted using water-soluble glues were cleaned effectively but the laser also affected the glue, causing it to crinkle and, on some occasions, the specimens were lost as the glue was unable to hold the specimen in place. Some specimens mounted with sticky carbon tabs were lost, as they had not been fixed down securely. Conodonts, because of their shape, proved particularly problematic as they only come into contact with the stub at one or two points and were often dislodged from the stub during treatment. A re-test using only conodonts showed that no damage was caused to the surface of the conodonts but only flat specimens able to be more securely fixed to the stub were cleaned without danger of being dislodged by the laser. All specimens mounted on inverted photographic paper were dislodged by the laser.

At the lowest fluence $\left(0.3 \mathrm{~J} \mathrm{~cm}^{-2}\right)$, none of the specimen's microscopic surface textures were affected by the laser, regardless of the wall structure type or composition, but the metallic surface coatings were still effectively removed (Plate 1). Increasing the fluence above $0.7 \mathrm{~J} \mathrm{~cm}^{-2}$ caused damage to the specimens and, above a fluence of $1.5 \mathrm{~J} \mathrm{~cm}^{-2}$, they were completely destroyed. At the lowest fluence, two cycles of coating and laser treatments had no affect on the specimens (Pl. 1, fig. 6). Fragments of dirt, mounting glue and residual carbon from the carbon tabs were removed along with the gold-palladium during treatments (Pl. 1, figs 2, 4).

\section{CONCLUSIONS}

1. A Q-Switched Nd: YAG (neodymium yttrium aluminium garnet) laser operating at $1064 \mathrm{~nm}$ (infrared), can remove gold-palladium coatings from micropalaeontological specimens mounted securely on sticky carbon tabs or in Araldite ${ }^{\circledR}$. This study shows that:

2. all microfossil types tested here can be treated, with the possible exception of specimens whose shape does not permit them to be mounted securely on stubs, for example conodonts;

3. this method has potential for removing other micropalaeontological coatings, such as gold, silver, aluminium, carbon, platinum and, possibly, waxes;

4. loose dirt is also removed from surfaces of specimens during treatment;

5. the process can be controlled easily as once the laser has been switched off, the cleaning process stops immediately, whereas damage can occur to specimens if left in sodium cyanide or ferric chloride for too long. Additionally, the chemical action may also continue for sometime after the sample has been removed from the reagent unless thorough and rigorous rinsing has been carried out;

6. the laser cleaning process is relatively quick - once specimens are prepared, up to ten specimens can be treated per minute;

7. the method is advantageous for health and safety reasons as toxic materials are avoided. However, correct health and safety measures for laser use should be followed. These are detailed in Cornish \& Jones (2002);

8. specimens are conserved because the energy is delivered as light so there is no mechanical contact with the object surface at a fluence of $0.3 \mathrm{~J} \mathrm{~cm}^{-2}$, thereby allowing the treatment of fragile surfaces and the reduction in risk of losing small specimens during cleaning.

\section{ACKNOWLEDGEMENTS}

Richard Hodgkinson (Scientific Associate, Natural History Museum) is thanked for discussions regarding micropalaeontological techniques. Drs Bob Jones (BP, Sunbury) and John Whittaker (Natural History Museum) provided identifications of the material from Dog's Bay, Ireland. The authors would like to thank Robert Waller (Canadian Museum of Nature) and 
New laser cleaning method

an anonymous reviewer for comments that improved the manuscript.

\section{Manuscript received 1 March 2004 \\ Manuscript accepted 10 July 2004}

\section{REFERENCES}

Cooper, M. 1998. Laser Cleaning in Conservation: an Introduction. Butterworth-Heinemann, Oxford.

Cornish, L. \& Jones, C. 2002. Laser cleaning natural history specimens and subsequent SEM examination. Conservation Science 2002. 101-106. Archetype, 278pp.
Crissman, R.S. \& McCann, P. 1979. A technique to remove goldpalladium from SEM samples. Micron, 10: 37-38.

Golden, J. 1989. Golden oldies: Curating SEM specimens. Collection Forum, 5 (1): 17-26.

Green, O.R. 2001. A manual of practical laboratory and field techniques in Palaeobiology. Kluwer Academic Publishers, Dordrecht, 538pp.

Hodgkinson, R.L. 1991. Microfossil processing: a damage report. Micropaleontology, 37 (3): 320-326.

Sela, J. \& Boyde, A. 1977. Cyanide removal of gold from SEM specimens. Journal of Microscopy, 111: 229-231. 